\title{
Erratum to: Embolisation of prostatic arteries as treatment of moderate to severe lower urinary symptoms (LUTS) secondary to benign hyperplasia: results of short- and mid-term follow-up
}

\author{
Joao Martins Pisco • Hugo Rio Tinto • Luís Campos Pinheiro • \\ Tiago Bilhim • Marisa Duarte • Lúcia Fernandes • \\ José Pereira • António G. Oliveira \\ Received: 25 April 2013 / Accepted: 26 April 2013 /Published online: 20 July 2013 \\ (C) European Society of Radiology 2013
}

\section{Erratum to: Eur Radiol}

DOI 10.1007/s00330-012-2714-9

\section{Statement from the Editor-in-Chief}

Since the online publication of this paper, a reader has drawn attention to the similarity between this paper and papers by the same authors in Techniques in Vascular and Interventional Radiology,

The online version of the original article can be found at http://dx.doi.org/ 10.1007/s00330-012-2714-9.

J. M. Pisco $\cdot$ H. Rio Tinto $\cdot$ T. Bilhim $\cdot$ M. Duarte $\cdot$ L. Fernandes Interventional Radiology, Saint Louis Hospital, Lisbon, Portugal

H. Rio Tinto $\cdot$ T. Bilhim $\cdot$ L. Fernandes

Radiology Department, Hospital de São José, Lisbon, Portugal

H. Rio Tinto $(\varangle) \cdot$ T. Bilhim

Radiology Department, Faculdade de Ciências Médicas,

Universidade Nova de Lisboa, Lisbon, Portugal

e-mail: hugo.tinto@gmail.com

L. Campos Pinheiro

Urology Department, Hospital de São José, Lisbon, Portugal

L. Campos Pinheiro

Urology Department, Faculdade de Ciências Médicas,

Universidade Nova de Lisboa, Lisbon, Portugal

\section{J. Pereira}

Radiology Department, Hospital Santo António dos Capuchos, Lisbon, Portugal

J. Pereira

Saint Louis Hospital, Interventional Radiology, Lisbon, Portugal

A. G. Oliveira

Biostatistics Department, Faculdade de Ciências Médicas,

Universidade Nova de Lisboa, Lisbon, Portugal
Rio Tinto H, Martins Pisco J, Bilhim T, Duarte M, Fernandes L, Pereira J, Campos Pinheiro L (2012) Prostatic artery embolization in the treatment of benign prostatic hyperplasia: short and medium follow-up. Tech Vasc Interv Radiol. 2012 Dec;15(4):290-3. doi: 10.1053/j.tvir.2012.09.005

and a recent paper in Radiology,

Pisco J, Campos Pinheiro L, Bilhim T, Duarte M, Rio Tinto H, Fernandes L, Vaz Santos V, Oliveira AG (2013) Prostatic arterial embolization for benign prostatic hyperplasia: short- and intermediate-term results. Radiology. 2013 Feb;266(2):668-77. doi: 10.1148/radiol.12111601. Epub 2012 Nov 30.

Because of this enquiry, the three editors of these journals have conferred and there are several difficulties related to potential redundant publication. The International Committee of Medical Journal Editors (ICMJE) has issued fairly robust criteria on this topic. A software cross-check reveals extensive overlap, with a high similarity index for the Techniques in Vascular and Interventional Radiology paper and the lowest for the European Radiology paper.

In their submission letter to European Radiology in August 2012 the authors answered, in response to question 'Have any of your study subjects or cohorts been previously reported?': 'NO'. That answer seemed unlikely as all three papers reported start and end dates in the March 2009 and April 2012 window.

When questioned, the authors claim that, as non-native English speakers, they misunderstood this question and that they considered the question just concerned each population; and thus they considered that the population was different in each paper. However they now admit that some subjects were previously reported in the Radiology paper. They now wish to publish the following retrospective acknowledgement: 
The preliminary results of our work were first published in Techniques in Vascular and Interventional Radiology. The medium-term follow-up in Radiology, and now with more patients and a longer follow-up in European Radiology.

There are some similar aspects in those three papers because the procedure, technique, follow-up and results were evaluated the same way.

Nevertheless there are significant differences that we should point out: these three papers have different time periods. All studies started in March 2009 when we also started to perform PAE. The end date for each paper is different: April 2011 for Radiology, June 2011 for Techniques in Vascular and Interventional Radiology and April 2012 for European Radiology. Apart of that there are some important differences concerning the exclusion criteria considered. Exclusion criteria were only malignancy and advanced atherosclerosis (Radiology). Later, other criteria such as detrusor failure, neurogenic bladder, urethral stenosis, bladder diverticula or stone were included (Techniques in Vascular and Interventional Radiology and European Radiology). Initially the QoL in inclusion criteria was $>3$ (Radiology and Techniques in Vascular and Interventional Radiology); however, later it was changed to $\geq 3$ (European Radiology).The inclusion criteria also changed. Initially men older than 50 years (Radiology and Techniques in Vascular and Interventional Radiology) were considered for this procedure, while later 45 years was the minimum (European Radiology).

Concerning the paper in Techniques in Vascular and Interventional Radiology, that specific paper was considered a review paper and neither retrospective or prospective.

Despite this above acknowledgement, these multiple submissions contravene correct publishing procedures as outlined by the ICMJE. Each paper should have carried a prospective acknowledgement to the material published in or submitted to the other journals, and this is now partially rectified in this erratum. Obviously it is important that this erratum is published so that future research workers possibly considering meta-analyses on this topic do not include all three articles as three different articles reporting on three different patient groups. All three editors have liaised about this topic and consider it is very important to remind authors that full acknowledgements of previously published or contemporaneously submitted work is essential at the time of submission.

The European Radiology paper comprises the largest group with the longest follow-up. Thus, it should probably be the single paper that any potential future meta-analysis should embrace.
Adrian K Dixon
Editor-in-Chief
European Radiology 